\title{
Lattice QCD on a novel vector architecture
}

\section{Benjamin Huth, Nils Meyer, Tilo Wettig*}

Department of Physics, University of Regensburg, 93040 Regensburg, Germany

E-mail: benjamin. huth@ur. de, nils.meyerdur.de, tilo.wettig@ur.de

\begin{abstract}
The SX-Aurora TSUBASA PCIe accelerator card is the newest model of NEC's SX architecture family. Its multi-core vector processor features a vector length of $16 \mathrm{kbits}$ and interfaces with up to 48 GB of HBM2 memory in the current models, available since 2018. The compute performance is up to $2.45 \mathrm{TFlop} / \mathrm{s}$ peak in double precision, and the memory throughput is up to $1.2 \mathrm{~TB} / \mathrm{s}$ peak. New models with improved performance characteristics are announced for the near future. In this contribution we discuss key aspects of the SX-Aurora and describe how we enabled the architecture in the Grid Lattice QCD framework.
\end{abstract}

The 37th Annual International Symposium on Lattice Field Theory - Lattice2019

16-22 June 2019

Wuhan, China

${ }^{*}$ Speaker. 


\section{Introduction}

Grid [1] is a modern Lattice QCD framework targeting parallel architectures. Architecturespecific code is confined to a few header files. The CPU implementations use compiler builtin functions (a.k.a. intrinsics) and assembly. There is also a generic, architecture-independent implementation based on $\mathrm{C} / \mathrm{C}++$ that relies on auto-vectorization.

Mainline Grid is limited to a vector register size of at most 512 bits. Here, we consider a new architecture with 16-kbit vector registers. We describe how we modified Grid to enable larger vector lengths and present initial performance benchmarks.

\section{NEC SX-Aurora TSUBASA}

\subsection{Overview}

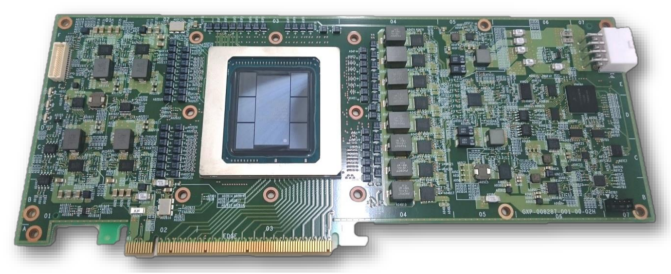

Figure 1: NEC SX-Aurora TSUBASA PCIe accelerator card (type 10). Picture published with permission from NEC, (C) by NEC.

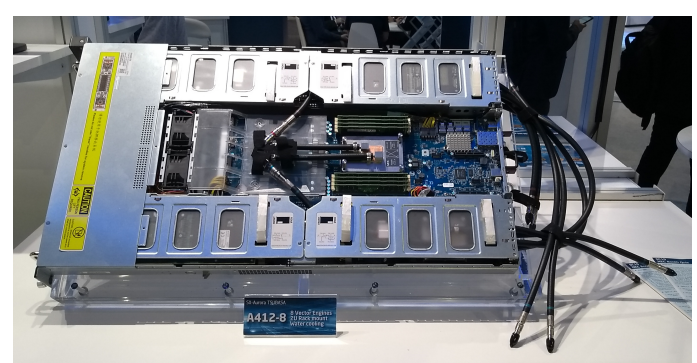

Figure 2: Liquid-cooled NEC A412-8 server presented at SC 19, featuring 8 SX-Aurora cards of novel type $10 \mathrm{E}$ attached to a singlesocket AMD Rome host CPU and fitting in $2 \mathrm{U}$.

The SX-Aurora TSUBASA, also called vector engine (VE), is the newest member of NEC's SX series [2]. In contrast to former vector supercomputer architectures, the SX-Aurora is designed as an accelerator card, see Fig. 1. At present it is available with PCIe Gen3 x16 interconnect (VE type 10). The accelerator hosts a vector processor with 8 cores. The card ships in 3 models, which we list in Table 1. For instance, the type 10A flagship model clocks at $1.6 \mathrm{GHz}$ and delivers 2.45 TFlop/s DP peak. The High Bandwidth Memory (HBM2) capacity is 48 GB with a throughput of $1.2 \mathrm{~TB} / \mathrm{s}$ peak. Improved accelerator models with higher main memory throughput (type 10E, type 20) and 10 cores (type 20) have been announced [3, 4].

Multiple SX-Aurora platforms are available, including workstation, rack-mounted server and supercomputer [2]. Up to 64 vector engines interconnected by InfiniBand fit into one A500 rack, delivering 157 TFlop/s DP peak. In Fig. 2 we show the novel A412-8 server presented at SC 19.

\begin{tabular}{l|rrr} 
Vector engine model & Type 10A & Type 10B & Type 10C \\
\hline \hline Clock frequency [GHz] & 1.6 & 1.4 & 1.4 \\
SP/DP peak performance [TFlop/s] & $4.91 / 2.45$ & $4.30 / 2.15$ & $4.30 / 2.15$ \\
HBM2 capacity [GB] & 48 & 48 & 24 \\
Memory throughput [TB/s] & 1.20 & 1.20 & 0.75
\end{tabular}

Table 1: NEC SX-Aurora TSUBASA type 10 models. 


\subsection{Vector engine architecture}

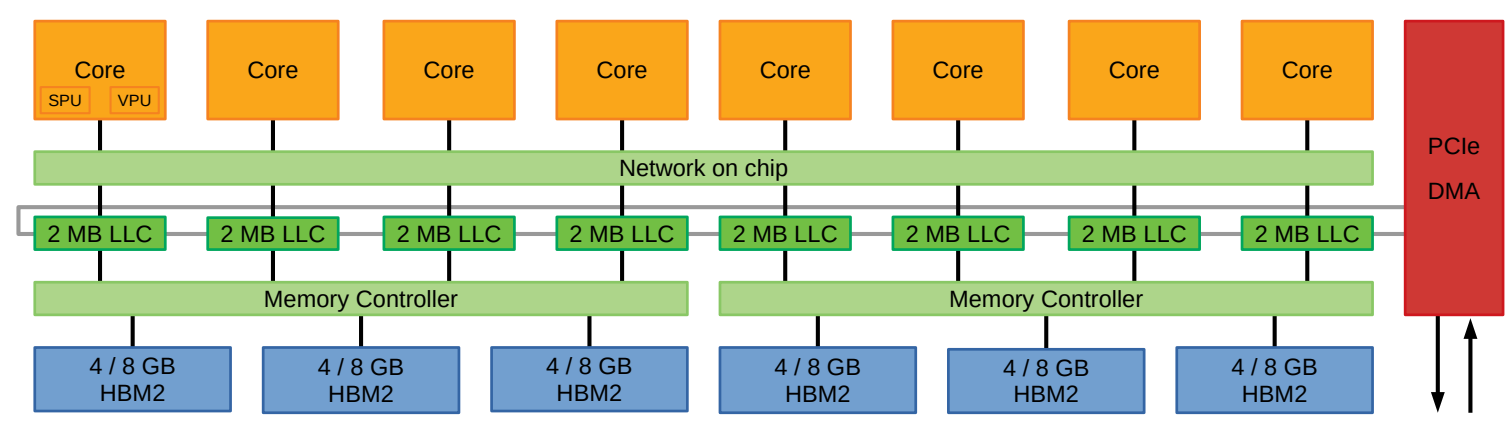

Figure 3: High-level architecture of the SX-Aurora type 10.

The high-level architecture of the SX-Aurora type 10 is shown in Fig. 3 [5, 6]. The chip contains 8 identical single-thread out-of-order cores. Each core comprises a scalar processing unit (SPU) with $32 \mathrm{kB} \mathrm{L1}$ cache and $256 \mathrm{kB} \mathrm{L} 2$ cache as well as a vector processing unit (VPU). The VPU processes (optionally masked) 16-kbit vector registers (corresponding to 256 real DP numbers) in 8 chunks of 2 kbits each.

There are 8 blocks of 2 MB last-level cache (LLC) connected by a $2 \mathrm{~d}$ network on chip. The VPUs directly access this (coherent) LLC. Two groups of 4 LLCs are connected to one memory controller each. Every controller addresses 3 stacks of HBM2. A ring bus interconnects the LLCs and allows for direct memory access (DMA) and PCIe traffic.

\subsection{Programming models and software stack}

The SX-Aurora does not run an operating system. It supports multiple modes of operation described in [7]. Most attractive to us is the VE execution mode: all application code is run on the accelerator, while system calls are directed to and executed on the host CPU.

Two C/C++ VE compilers by NEC are available: ncc (closed source) and clang/LLVM VE (open source). The latter is still in an early development stage. Both compilers support OpenMP for thread-level parallelization but differ in how they vectorize. ncc exclusively relies on autovectorization and does not support intrinsics, while clang/LLVM VE currently does not support auto-vectorization and relies on intrinsics instead.

The NEC software stack also includes support for MPI, debugging, profiling (ncc only) and optimized math libraries, e.g., BLAS and LAPACK.

\section{Grid on the NEC SX-Aurora}

\subsection{Enabling larger vector lengths in Grid}

Grid decomposes the lattice into one-dimensional arrays (which we shall call Gridarrays in the following) that usually have the same size as the architecture's vector registers. Operations on Gridarrays can achieve 100\% SIMD efficiency.

On CPUs, mainline Grid is restricted by design to Gridarrays of at most 512 bits. This restriction does not appear directly in the code. Rather, it is an assumption in shift and stencil operations. 
To explain the origin of this restriction, it is helpful to understand how the lattice sites are mapped to Gridarrays. We first introduce some notation.

- We assume a $d$-dimensional lattice with $V=L_{0} \cdot \ldots \cdot L_{d-1}$ lattice sites.

- The degrees of freedom of a single lattice site are given by $m$ numbers. We assume that the data type of these numbers is fixed (e.g., single real, single complex, etc.).

- A Gridarray contains $n$ numbers of this fixed data type. ${ }^{1}$

- Grid defines an array of $d$ integers $\left\{n_{0}, \ldots, n_{d-1}\right\}$ called "SIMD layout". These integers are powers of 2 and have to satisfy the condition $n_{0} \cdot \ldots \cdot n_{d-1}=n$.

The mapping then proceeds as follows.

1. The lattice is decomposed into sublattices containing $n$ lattice sites each. The number $n_{i}$ $(i=0, \ldots, d-1)$ equals the number of sublattice sites in dimension $i$. Note that Grid performs the decomposition in such a way that adjacent sites of the full lattice are mapped to different sublattices. In fact, the sites of a given sublattice are as far away from one another in the full lattice as possible (see also Fig. 4).

2. A given sublattice is mapped onto $m$ Gridarrays. One Gridarray contains one of the $m$ degrees of freedom of all $n$ sublattice sites (see Fig. 4 for $m=1$ with real numbers).

We first consider a single Grid process (without MPI communication). Given the decomposition just described, shifts can be done by a few simple methods (see Fig. 4, cases A and B):

- If no site of a sublattice is part of the lattice boundary in the shift direction, a simple copy of all involved Gridarrays is sufficient.

- Otherwise, in addition to the copy, the elements within a Gridarray must be rearranged. Mainline Grid can handle two cases:

- Case A: A SIMD layout with all entries but one equal to 1. Then the Grid function rotate performs the rearrangement.

- Case B: Any other SIMD layout than case A, with the restriction that no entry is larger than 2 . Then the Grid function permute rearranges the sites in the right way.

Other SIMD layouts are not supported by mainline Grid, and thus the maximum SIMD layout for a 4-dimensional lattice is $\{2,2,2,2\}$. For a lattice with SP real numbers this corresponds to a maximum vector register size of 512 bits, which explains the restriction mentioned above. However, the SX-Aurora with its $16-\mathrm{kbit}$ vector registers requires at least $\{2,4,4,4\}$ (for a DP complex lattice). Therefore, an extension of the shift and stencil algorithms is necessary.

The new transformation should have the functionality of rotate and permute, but for an arbitrary SIMD layout. Furthermore, this operation should be vectorizeable and have similar performance. The new function split_rotate (shown in the following for the double-precision case) fulfills these aims:

\footnotetext{
${ }^{1}$ For example, if the Gridarray size is 512 bits, we have $n=4$ for double complex, $n=8$ for double real or single complex, and $n=16$ for single real.
} 

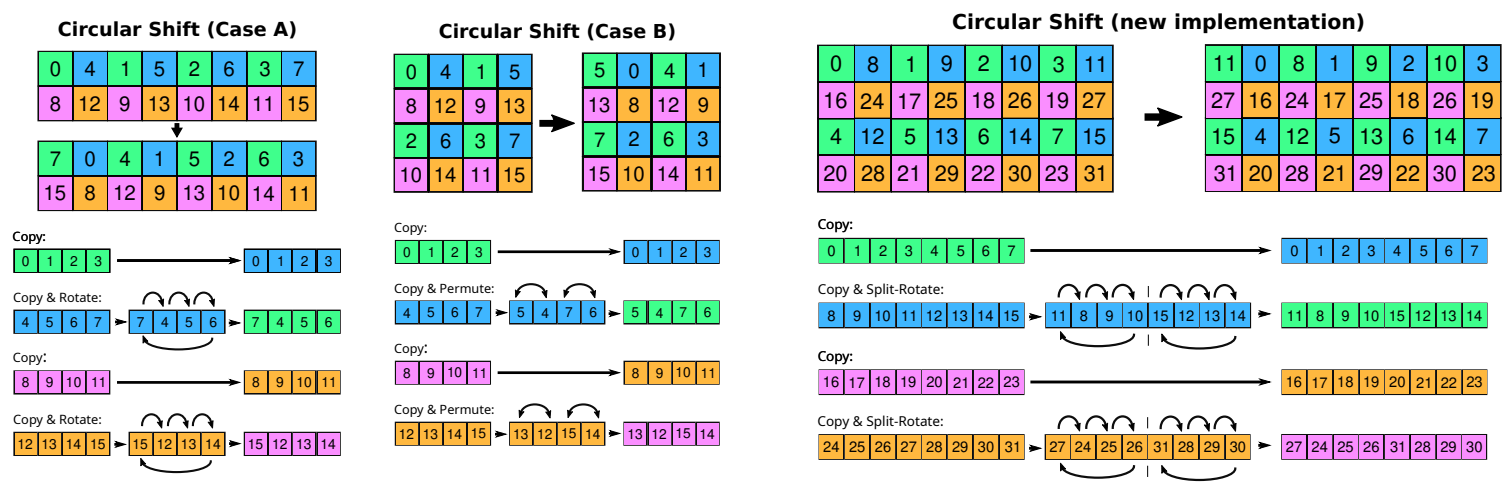

Figure 4: Examples for shifts in a 2d lattice with $m=1$. Case A (rotate) has SIMD layout $\{4,1\}$, case B (permute) $\{2,2\}$. In both cases $n=4$. The new implementation split_rotate (right-most figure, $n=8$ ) is able to handle a SIMD layout of, e.g., $\{4,2\}$. The colored boxes denote positions in memory, where all boxes with the same color are in contiguous memory. At the bottom we display the transformations of the underlying Gridarrays.

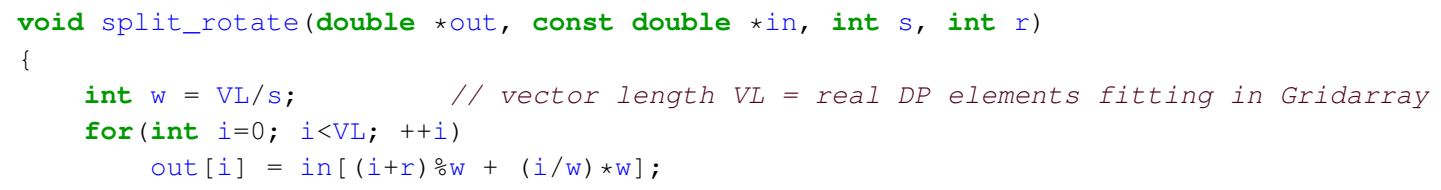

The split parameter $s$ specifies into how many subarrays the Gridarray is split. Then these subarrays are rotated by $r$. In the case of complex numbers, the input $r$ must be multiplied by 2 . For $s=1$, we obtain mainline Grid's rotate function. The effect of split_rotate on a $2 \mathrm{~d}$ lattice is shown in Fig. 4. Examples for shifts on a 3d sublattice by split_rotate are shown in Fig. 5.

We have replaced rotate and permute by split_rotate in shift and stencil operations and thereby enabled Grid to handle Gridarray sizes of $128 \times 2^{k}$ bits (with $k \in \mathbb{N}$ ). Our implementation of split_rotate is done in generic $\mathrm{C} / \mathrm{C}++$ code, which is architecture-independent and thus applicable beyond the SX-Aurora.

The algorithm described above works for a single Grid process. When using Grid on multiple nodes (with MPI communication), some Gridarrays have to be broken up and partially transferred to the neighboring node in order to perform a lattice shift. In mainline Grid, this is also restricted to the same SIMD layouts as described above. We have enabled the required functionality for larger Gridarray sizes of $128 \times 2^{k}$ bits. However, the implementation still needs to be optimized.

\subsection{Status of porting Grid}

For Grid we have chosen the VE execution model, relying mainly on the ncc compiler and its auto-vectorization capabilities. The progress of porting was slowed down by some compiler and toolchain issues (e.g., Grid compiled with OpenMP only since the summer of 2019). Whereas the issue of enabling larger vector lengths is resolved, the performance of Grid still needs to be tuned. Full MPI support is under ongoing development. We also experimented with Grid and the intrinsics of the clang/LLVM VE compiler, but this option will only become viable once the compiler matures. All sources are available at [8], where we forked Grid version 0.8.2. 

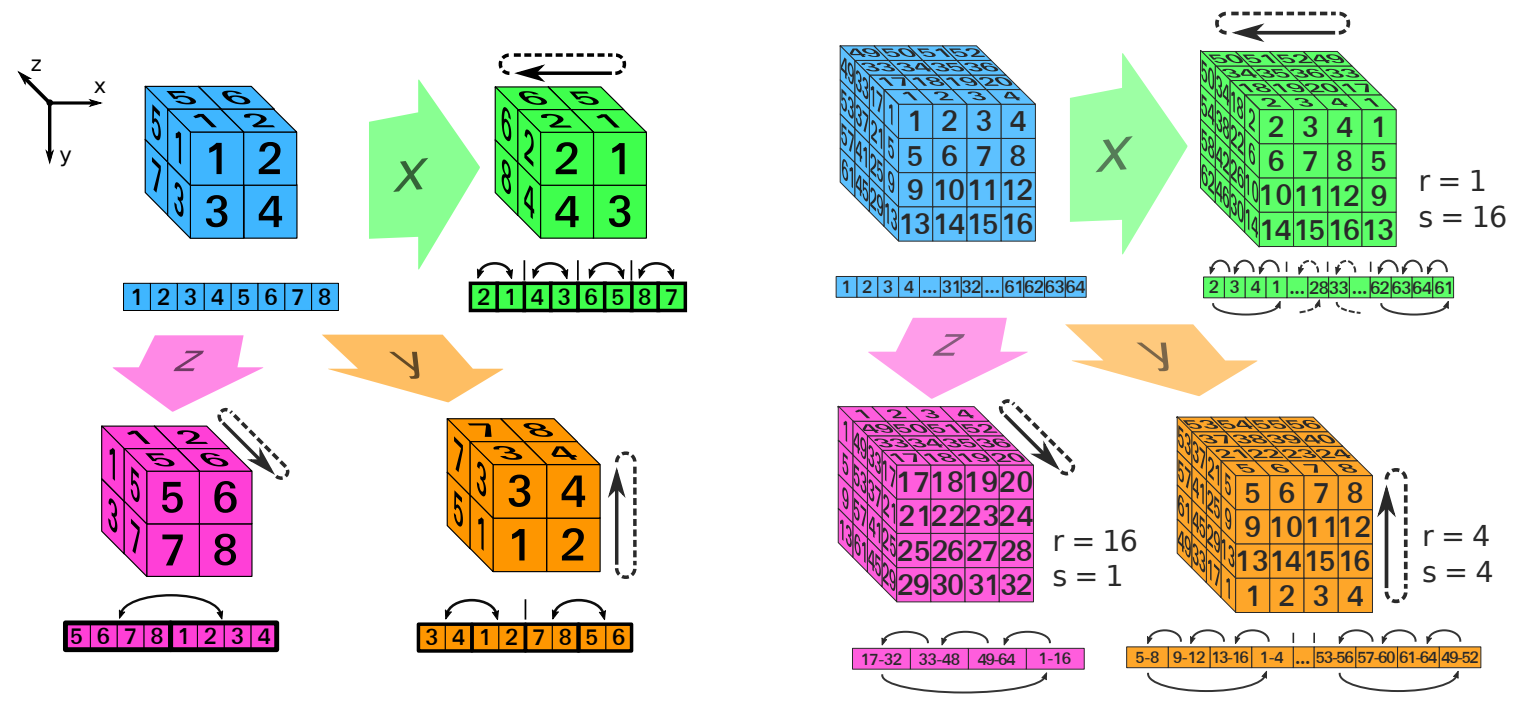

Figure 5: Shifts on a $3 \mathrm{~d}$ sublattice with $m=1$. Left: A small sublattice with side length $=2$ and SIMD layout $\{2,2,2\}$ is shifted by the permute function. Right: A larger sublattice with side length $=4$ and SIMD layout $\{4,4,4\}$ is shifted using the split_rotate function. The corresponding parameters $s$ and $r$ are also shown.

\subsection{Preliminary performance results}

In Fig. 6a we show how the size of the Gridarrays affects the performance of a custom implementation of SU(3) matrix-matrix multiplication. As in mainline Grid, the data layout in memory is an array of two-element structures $\{\mathrm{re}, \mathrm{im}\}$. Best performance is achieved using clang/LLVM VE intrinsics and when the Gridarray size is twice the register size $(2 \cdot 16 \mathrm{kbit})$. The SX-Aurora supports a strided load instruction, which is applied twice to load the real and imaginary parts into separate registers (strided store is analoguous). ncc also uses strided load/store, but the performance is significantly worse due to superfluous copies between register file and LLC.

Figures $6 \mathrm{c}$ and $6 \mathrm{~b}$ show SU(3) matrix-matrix multiplication (custom implementation vs Grid, both $100 \%$ SIMD efficient) for two different scaling scenarios: increasing lattice volume using a single thread, and strong thread scaling at constant volume. Again, the performance of clang/LLVM VE intrinsics is significantly better than auto-vectorization by ncc due to the superfluous copies mentioned above. For comparison we also show the performance of the Intel KNL 7210 in Fig. 6b.

Figure $6 \mathrm{~d}$ compares the performance of both shift implementations on a platform where both functions can be called, here Intel KNL 7210 with vector length 512 bits. In the generic version of the Wilson kernel, split_rotate performs slightly better than permute. Both are surpassed by the hand-unrolled version using permute, which we did not implement for split_rotate.

\section{Summary and outlook}

We have shown how to modify Grid to deal with larger vector register sizes than the current 512-bit limit and presented performance benchmarks on the SX-Aurora. Work on MPI support is in progress. Once this support is available and further performance optimizations are implemented, the SX-Aurora will be an interesting option for Lattice QCD. 
Single-thread SU(3) MM multiplication (custom implementation, w/o OpenMP)

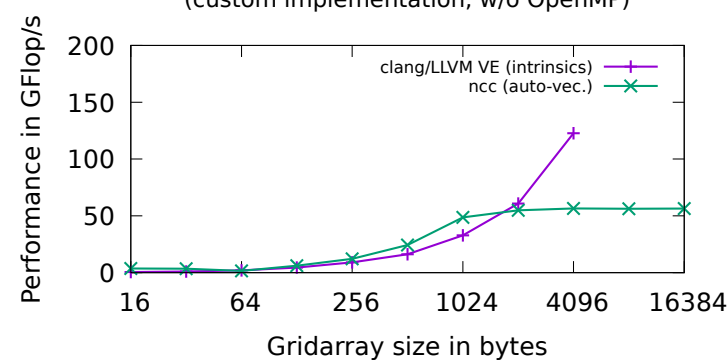

(a) Single-thread custom SU(3) matrix-matrix multiplication without OpenMP, scaling up the size of the Gridarray: intrinsics vs auto-vectorization.

Multi-thread SU(3) MM multiplication (custom vs Grid, w/ OpenMP, V=404)

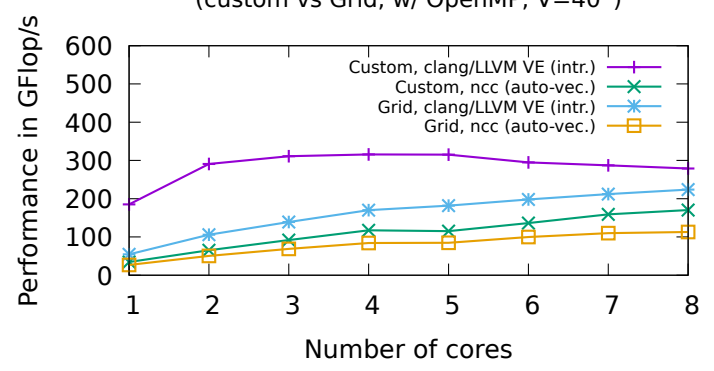

(c) Multi-thread SU(3) matrix-matrix multiplication using OpenMP: intrinsics vs auto-vectorization, custom vs Grid.
Single-thread SU(3) MM multiplication (custom vs Grid, w/o OpenMP)

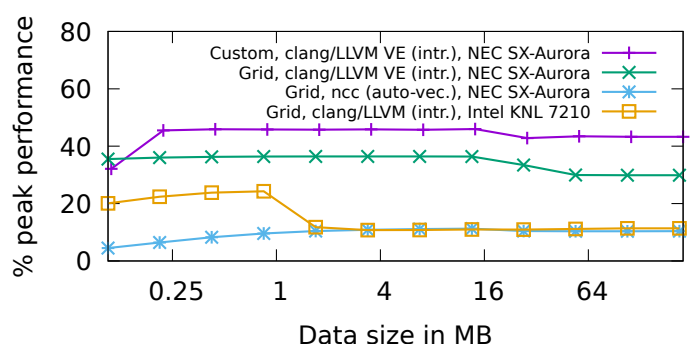

(b) Single-thread SU(3) matrix-matrix multiplication without OpenMP, increasing the lattice size: intrinsics vs auto-vectorization, custom vs Grid.

Wilson Dirac operator on Intel KNL 7210 (mainline Grid vs split_rotate, w/OpenMP)

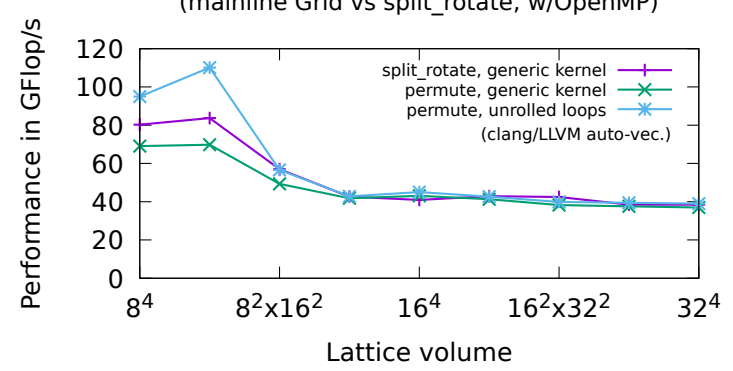

(d) Application of the Wilson Dirac operator on KNL with OpenMP: mainline Grid implementation (in this case, permute only) vs split_rotate.

Figure 6: Preliminary performance benchmarks. The SX-Aurora benchmarks were performed on a type 10B card using ncc 2.5.1 and clang/LLVM VE 10.0.0. For KNL we used clang/LLVM 5.0.0.

\section{Acknowledgment}

This work was supported by DFG in the framework of SFB/TRR 55 (project QPACE 4). We thank Erlangen University, Germany, and KEK, Japan, for access to the SX-Aurora and for support.

\section{References}

[1] P. Boyle, A. Yamaguchi, G. Cossu and A. Portelli, PoS (LATTICE 2015) 023 [1512 . 03487 ]

[2] https://www.nec.com/en/global/solutions/hpc/sx

[3] S. Momose, 2nd Aurora Deep Dive Workshop at RWTH Aachen University (2019)

[4] https://fuse.wikichip.org/news/3073/nec-refreshes-sx-aurora-vector-engine-outlines-roadmap

[5] Y. Yamada and S. Momose, Hot Chips (2018)

[6] https://en.wikichip.org/wiki/nec/microarchitectures/sx-aurora

[7] K. Komatsu et al., Proceedings of SC 18 (2018) 54

[8] B. Huth, “Grid fork SX-Aurora”, https://github.com/benjaminhuth/Grid 